\title{
Increased microRNA-30a levels in bronchoalveolar lavage fluid as a diagnostic biomarker for lung cancer
}

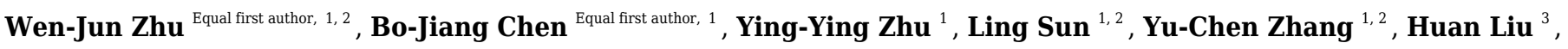 \\ Feng-Ming Luo ${ }^{\text {Corresp. } 1,2}$ \\ ${ }^{1}$ Department of Respiratory and Critical Care Medicine, West China Hospital, Sichuan University, Chengdu, China \\ 2 Laboratory of Pulmonary Immunology and Inflammation, Frontiers Science Center for Disease-related Molecular Network, Sichuan University, Chengdu, \\ Sichuan, China \\ 3 Department of General Medicine, University-Town Hospital of Chongqing Medical University, Chongqing, China \\ Corresponding Author: Feng-Ming Luo \\ Email address: fengmingluo@outlook.com
}

Background: MicroRNA-30a (miRNA-30a) levels have been shown to increase in the plasma of lung cancer patients. Herein, we evaluated the miRNA-30a levels in the bronchoalveolar lavage fluid (BALF) of lung cancer patients as a potential biomarker for lung cancer diagnosis.

Methods: BALF miRNA-30a expression of 174 subjects was quantified using quantitative real-time reverse transcription-polymerase chain reaction and compared between lung cancer patients and control patients with benign lung diseases. Moreover, its diagnostic value was evaluated by performing receiver operating characteristic (ROC) curve analysis.

Results: The relative BALF miRNA-30a expression was significantly higher in the lung cancer patients than in the controls $(0.74 \pm 0.55$ versus $0.07 \pm 0.48$, respectively, $p<0.001)$ as well as in lung cancer patients with stage I-IIA disease than in those with stage IIB-IV disease $(0.98 \pm 0.64$ versus $0.66 \pm 0.54$, respectively, $p<0.05)$. Additionally, miRNA-30a distinguished benign lung diseases from lung cancers, with an area under the ROC curve (AUC) of 0.822 . ROC analysis also revealed an AUC of 0.875 for the Youden index-based optimal cut-off points for stage I-IIA adenocarcinoma. Thus, increased miRNA-30a levels in BALF may be a useful biomarker for non-small-cell lung cancer diagnosis. 
1 RESEARCH ARTICLE

2 Increased microRNA-30a levels in bronchoalveolar lavage

3 fluid as a diagnostic biomarker for lung cancer

4

5 Wen-Jun Zhu ${ }^{1,2 \#}$, Bo-Jiang Chen ${ }^{1 \#}$, Ying-Ying Zhu ${ }^{1}$, Ling Sun ${ }^{1,2}$, Yu-Chen Zhang ${ }^{1,2}$, Huan Liu ${ }^{3}$,

$6 \quad$ Feng-Ming Luo $^{1,2 *}$

7

$8{ }^{1}$ Department of Respiratory and Critical Care Medicine, West China Hospital, Sichuan

9 University, Chengdu 610041, China

10 2Laboratory of Pulmonary Immunology and Inflammation, Frontiers Science Center for Disease-

\section{*Corresponding author}

Feng-Ming Luo

Fengming Luo, Department of Pulmonary and Critical Care Medicine, West China Hospital, Sichuan University, Chengdu 610041, Sichuan, China, Tel: +86-28-85422759, Fax: +86-2885422759,E-mail:fengmingluo@outlook.com

Abstract 
23 Background: MicroRNA-30a (miRNA-30a) levels have been shown to increase in the plasma of

24 lung cancer patients. Herein, we evaluated the miRNA-30a levels in the bronchoalveolar lavage

fluid (BALF) of lung cancer patients as a potential biomarker for lung cancer diagnosis.

Methods: BALF miRNA-30a expression of 174 subjects was quantified using quantitative realtime reverse transcription-polymerase chain reaction and compared between lung cancer patients and control patients with benign lung diseases. Moreover, its diagnostic value was evaluated by performing receiver operating characteristic (ROC) curve analysis.

Results: The relative BALF miRNA-30a expression was significantly higher in the lung cancer patients than in the controls $(0.74 \pm 0.55$ versus $0.07 \pm 0.48$, respectively, $\mathrm{p}<0.001)$ as well as in lung cancer patients with stage I-IIA disease than in those with stage IIB-IV disease $(0.98 \pm$ 0.64 versus $0.66 \pm 0.54$, respectively, $\mathrm{p}<0.05)$. Additionally, miRNA-30a distinguished benign lung diseases from lung cancers, with an area under the ROC curve (AUC) of 0.822. ROC analysis also revealed an AUC of 0.875 for the Youden index-based optimal cut-off points for stage I-IIA adenocarcinoma. Thus, increased miRNA-30a levels in BALF may be a useful biomarker for non-small-cell lung cancer diagnosis.

Keywords: miRNA-30a, lung cancer, bronchoalveolar lavage fluid (BALF), tumor marker, early diagnosis, biomarker 


\section{Introduction}

43 Lung cancer is the leading cause of cancer-related mortality worldwide (Torre et al. 2016). The

44 World Health Organization estimates that the trends of lung cancer deaths will continue to rise, 45 largely due to an increase in global tobacco use, especially in Asia (Dela Cruz et al. 2011). In 46 addition, lung cancer is usually diagnosed at an advanced stage because of the lack of clinical 47 symptoms (Tissot et al. 2015), leading a poor prognosis (Alberg et al. 2013). Given its low rate 48 of early diagnosis, lung cancer not only seriously affects the survival and prognosis of patients

Biopsy under flexible bronchoscopy (FB) is now established as an essential diagnostic tool for patients with abnormal imaging or sputum findings, according to the routine lung cancer screening process (Du Rand et al. 2013). The diagnostic yield from FB ranges from 48\% to 93\%, depending on whether the tumor is visible within the bronchial tree (Rivera \& Mehta 2007). However, biopsy with FB is limited when the lesions are with obviously exposed blood vessels and when patients with clinical risk factors for bleeding (such as anticoagulation therapy, renal dysfunction) (Du Rand et al. 2013). Bronchial washings with FB is an alternation when biopsy is limited, which increases the diagnostic yield of FB in patients with lung cancer (McLean et al. 1998). Therefore, alternative diagnostic process should be taken into account for individuals undergoing FB but with invisible tumor in the bronchial tree or risk factors of biopsy.

MicroRNAs (miRNAs) are a class of 22-nucleotide long, non-coding, single-stranded RNAs (Yi et al. 2003). There is increasing evidence that miRNA expression is associated with various 
65

66

67

84

human cancers and may function as tumor suppressors or oncogenes (Bridge et al. 2012).

MiRNAs play an important role in the cancer cells' development (Bussing et al. 2008; Johnson et al. 2007), invasion, metastasis(Yao et al. 2010), proliferation, differentiation, apoptosis, and neoplastic transformation(Bartel 2004) (Boehm \& Slack 2005), indicating that miRNAs may serve as a new source of biomarkers for the diagnosis of tumors (Chen et al. 2008; EsquelaKerscher \& Slack 2006; Fredsøe et al. 2018; Kim et al. 2015; Mitchell et al. 2008).

MiRNA-30a is a member of the miRNA-30 family, which contains five distinct premature miRNAs sequences (miR-30a, -30b, -30c, -30d and -30e)(Bridge et al. 2012; Mao et al. 2018). Increasing evidence suggested that circulating miRNA-30a has been correlated with several types of human cancers(Fredsøe et al. 2018; Zeng et al. 2013; Zhou et al. 2015). miRNA-30a has been found to be related to the transformation, prognosis, and diagnosis of lung cancer(Kumarswamy et al. 2012; Tang et al. 2015; Wen et al. 2015a), which indicates miRNA30a may act as a biomarker for lung cancer detection. Our previous study demonstrated that plasma miRNA-30a levels increased in non-small-cell lung cancer (NSCLC) with a relatively high value in the diagnosis of NSCLC(Sun et al. 2016). However, the expression level of miRNA-30a levels in bronchoalveolar lavage fluid (BALF) and diagnostic value of BALF miRNA-30a for those individuals suspected for lung cancer with indications of flexible bronchoscopy but with relatively high risk to perform biopsy still remains unknown.

In this study, we investigated the relative expression of miRNA-30a in BALF and explored its diagnostic value for lung cancer, especially at an early stage. 
88 Material \& Methods

$89 \quad$ Study setting and subjects

90 The protocol for this prospective study was approved by the Medical Ethics Committee of the

91 West China Hospital, Sichuan University (approval number 2018-83). Samples were collected

92 and processed in a double-blind manner. All patients were grouped based on the pathological

93 diagnosis after miRNA-30a levels were measured, and all other data were collected. By the end

94 of this study, 99 patients with histologically proven primary lung cancer, 55 patients with benign

95 lung diseases 20 healthy controls were recruited between December 2015 and February 2017 at

96 our institution. All the patients have not undergone any types of therapy before sample collection.

97

The clinical data were acquired for each subject from electronic medical records and included information regarding a complete history and physical examination, serological examination, diagnostic imaging scans, and pathology reports. Epidemiological data were also collected using a self-reported questionnaire, which included information such as demographics, performance status, occupational history, and previous medical history.

\section{Sample collection}

Written informed consent was obtained from all patients prior to bronchoscopy. Plasma and BALF collection, miRNA extraction and analysis were performed based on the same SOP (Standard Operating Procedure) for each patient. Briefly, fasting peripheral venous blood (5 ml) and BALF (8ml) were collected within an hour before and during bronchoscopy process respectively. For patient with lung cancer, BALF specimens were collected from both the sides of the chest lung, including where the tumor was located and the contralateral side (Kim et al. 
111 2015). The blood sample and BALF specimens were centrifuged immediately after collection, at

$1124^{\circ} \mathrm{C} 3,000 \mathrm{rpm} 10 \mathrm{~min}$ and $4^{\circ} \mathrm{C} 2,000 \mathrm{rpm} 10 \mathrm{~min}$ respectively. The supernatants were stored at

$1134^{\circ} \mathrm{C}$ temporally and were transferred to $-80^{\circ} \mathrm{C}$ within one hour. RNA sample extraction, Reverse

114 transcription (RT) Quantitative real-time polymerase chain reaction (qPCR) and ELISA

115 (enzyme-linked immunosorbent assay) procedure were performed within one week after BALF

116 and plasma sample collection.

117

118

RNA quality was measured by RNA concentration, purity and integrity. RNA concentration and

119 purity was detected by measuring the absorbance at $260 \mathrm{~nm} / 280 \mathrm{~nm}(\mathrm{~A} 260 / \mathrm{A} 280)$ and 260

120

nm/230 nm(A260/A230) in a Nanodrop 1000 spectrophotometer (Thermo Fisher Scientific Inc.,

DE, USA). RNA integrity was measured by 1\%AGE (Agarose Gel Electrophoresis). With the

122 quality of high purification(A260/A280 was in 1.8-2.1, A260/A230>1.8) and integrate bands, the RNA extracted is suitable for molecular biology operation.

\section{Measurement of miRNA-30a levels}

Total RNA was isolated from BALF specimens using the TRIzol-based method (Life

Technologies, Carlsbad, CA, USA), and the RNA pellet was dissolved in $30 \mu \mathrm{L}$ of RNAase-free water as previously reported (Sun et al. 2016). The purity and concentration of RNA were evaluated using a ScanDrop 100 spectrophotometer (Analytik Jena AG, Jena, Germany). Reverse transcription (RT) was performed using a RevertAid RT kit (Thermo Fisher Scientific, Waltham, MA, USA) according to the manufacturer's instructions.

132 Quantitative real-time polymerase chain reaction (RT-qPCR) assays were performed as 133 previously reported (Sun et al. 2016) in a $20 \mu \mathrm{L}$ volume using a CFX96 real-time PCR system 
134 (Bio-Rad, Hercules, CA, USA). The reactions were incubated at $95^{\circ} \mathrm{C}$ for $3 \mathrm{~min}$, followed by 40

135 cycles of $10 \mathrm{~s}$ at $95^{\circ} \mathrm{C}, 15 \mathrm{~s}$ at $59^{\circ} \mathrm{C}$, and $10 \mathrm{~s}$ at $72^{\circ} \mathrm{C}$. The relative expression levels of miRNA-

136 30a were calculated as expression value of miRNA-30a to the expression value of U6 via the

137 comparative $2^{-\Delta \Delta \mathrm{CT}}$ method (Fredsøe et al. 2018). Finally, the results were processed and

138 analyzed using the Bio-Rad CFX Manager.

Statistical analyses

All statistical analyses were performed using SPSS version 21 (SPSS Inc., Chicago, IL, USA).

The association of categorical variables was assessed using the chi-squared test. A $t$-test or

analysis of variance was used to explore the distribution of continuous variables among the study groups, as appropriate. Receiver operating characteristic (ROC) curve analysis was applied to investigate the potential of miRNA-30a and other tumour markers to distinguish between the benign and malignant groups. The optimal Youden index was used as the cut-off point for lung cancer diagnosis (Berezikov et al. 2006) based on the sensitivity and specificity. Values of $\mathrm{p}<0.05$ were considered to be statistically significant.

\section{Results}

\section{Clinical characteristics of study subjects}

152 BALF samples were collected from 99 patients with histologically proven lung cancer, 55 
157 System, 19, 8, 31, and 26 patients with stages I, II, III, and IV lung cancer, respectively, were

158 identified based on the postoperative pathology. Among patients with benign lung diseases, there

159 were 39 males and 16 females with a mean age of $54.53 \pm 10.62$ years, including 47 pathology-

160 verified cases of pneumonia, 2 cases of inflammatory pseudotumor, and 6 cases with benign lung

161 nodules. There were 12 males and 8 females in the healthy control group, and the mean age was

$16253.05 \pm 13.20$. Patients with benign lung diseases as well as patients in the healthy control group

163 had not developed any types of cancer during the whole follow-up period. There were no

164 statistically significant differences in the clinical characteristics among three groups (Table 1).

165

166

167

168

169

170

171

172

173

174

175

176

177

178

179

\section{BALF miRNA-30a levels in three groups}

MiRNA-30a was significantly upregulated in BALF specimens collected from the lesion side of lung cancer patients compared with patients with lung benign lesions and healthy controls (all, $\mathrm{p}<0.001$ ) (Fig. 1).

\section{MiRNA-30a levels in BALF specimens obtained from the sides with and without lung cancer}

\section{lesions}

The origin of miRNA-30a in BALF is unknown. It may be secreted by tumour cells, cells adjacent to tumour tissues, immune cells accumulated around the tumour, or even cells circulating in the bloodstream. It is reasonable to hypothesize that the level of miRNA-30a in BALF specimens obtained from the side with the lesion would be higher than in that obtained from the side without the lesion. Therefore, BALF specimens were collected from lung cancer patients via flexible bronchoscopy from both the sides, with and without the lesions, simultaneously. No statistically significant difference was found in the BALF miRNA-30a levels 
180 between the two sides $(0.23 \pm 0.65, \mathrm{p}=0.06)$ (Fig.2). Therefore, we subsequently analyzed the

181 level of miRNA-30a in the BALF specimens obtained from the side with the lung cancer lesion.

182

183

184

185

186

187

188

189

190

191

192

193

194

195

196

197

198

199

200

201

202

Correlation between BALF miRNA-30a levels and clinicopathological features of lung cancer

To explore the possible relationships between BALF miRNA-30a levels and the

clinicopathological features of lung cancer, we analyzed the data based on the following factors:

age, sex, smoking status, histological type, and TNM stage. No statistically significant

differences were found between miRNA-30a levels and these clinicopathological features, apart from the clinical TNM stage (Table 2 and Fig. 3).

\section{Diagnostic value of miRNA-30a levels in lung cancer patients}

Since the level of miRNA-30a in BALF specimens was significantly higher among lung cancer patients compared with that of the controls, miRNA-30a might serve as a biomarker for lung cancer diagnosis. ROC curve analysis was performed to evaluate the diagnostic value of miRNA-30a levels. The area under the curve (AUC) for miRNA-30a was $0.822(95 \%$ confidence interval [CI]: 0.752-0.892, p<0.001) (Fig. 4).

As shown in Table 3, the best diagnostic cut-off value of relative miRNA-30a levels in BALF was 2.0316 (with a diagnostic sensitivity and specificity of $80.8 \%$ and $69.1 \%$, respectively).

\section{Diagnostic value of BALF miRNA-30a level for stages I-IIA lung cancer and benign lung} disease

Compared with levels among patients with benign lung diseases, miRNA-30a was significantly upregulated in the BALF of patients with stages I-IIA lung cancer $(0.98 \pm 0.64$ versus $0.07 \pm$ 
203 0.48, respectively, p<0.001) (Fig. 5), with an AUC of 0.882 (95\% CI: 0.806-0.958, p<0.001)

204 (Fig. 6). The best diagnostic cut-off value of relative miRNA-30a levels in BALF was 2.6508

205 (with a diagnostic sensitivity and specificity of $90.9 \%$ and $74.5 \%$, respectively) (Table 4). This

206 indicated that the upregulated BALF miRNA-30a expression level could be used as an early

207 diagnostic marker for lung cancer.

208

209

Diagnostic value of miRNA-30a expression in BALF among different pathologic types and

\section{TNM stages of lung cancer and benign lung disease}

211 There were statistically significant differences in BALF miRNA-30a levels among different

212 pathologic types and TNM stages of lung cancer and benign lung disease $(\mathrm{p}<0.001)$. ROC

213 analysis revealed an AUC of 0.875 for the Youden index-based optimal cut-off points for stages

214 I-IIA adenocarcinoma (Fig. 7). Thus, increased levels of miRNA-30a in BALF may serve as a 215 diagnostic biomarker for stage I-IIA adenocarcinoma (Tables 5 and 6).

216

Combined diagnostic value of BALF miRNA-30a and plasma tumour markers

218 In addition, we compared the diagnostic value of BALF miRNA-30a with that of traditional plasma tumour markers (carcinoembryonic antigen [CEA] and CYFRA21-1) based on the ability to differentiate lung cancer patients from patients with benign lung disease. We determined the BALF miRNA-30a, plasma CEA, and CYFRA21-1 levels in stages I-IIA NSCLC and control groups and found that the AUC of miRNA-30a was larger than that of CEA and CYFRA21-1. A combination of BALF miRNA-30a levels with plasma CEA and CYFRA21-1 levels could improve the diagnostic value for advanced NSCLC. When plasma CYFRA21-1, CEA, and 
226

227

228

229

230

231

232

233

234

235

236

237

238

239

240

241

242

243

244

245

246

247 248

stages I-IIA NSCLC and $92.3 \%$ for stages IIB-IV NSCLC, which makes this combination preferable for the diagnosis of NSCLC (Table 7 and Fig. 8).

\section{Discussion}

Our previous data showed miRNA-30a was down-regulated and up-regulated in lung cancer tissue(Sun et al. 2016) and plasma respectively (Liang et al. 2019), which arouse our interest in analyzing miRNA-30a expression in BALF of lung cancer. In this hospital-based case-control study, we found the expression level of miRNA-30a in BALF was significantly up-regulated in lung cancer patients when both compared to those with benign lung disease and healthy control patients (both $\mathrm{p}<0.001$ ). The expression level of miRNA-30a in BALF was significantly higher in individuals with early stage (stage I-IIA) of lung cancer when compared to those with stage IIB-IV ( $\mathrm{p}<0.05)$. The ROC curve analysis revealed that the BALF miRNA-30a level could well distinguish lung benign disease and lung cancer as well as early stage of lung cancer with the relative high AUC of 0.822 and 0.882 respectively. The diagnostic value was even higher when combined BALF miRNA-30a, CEA and CYFRA21-1. All those findings indicated that BALF miRNA-30a relative expression level could be used as a biomarker in early detection of lung cancer for individuals undergoing FB but with invisible tumor in the bronchial tree or risk factors of biopsy.

Abundant circulation miRNAs have been demonstrated to play a vital role in early cancer detection (Kim et al. 2015; Mitchell et al. 2008; Tsujiura et al. 2010). The circulating cell-free miRNAs have shown great promise as a new class of cancer biomarkers, owing to their surprisingly high stability in body fluids, association with disease states, and ease of 
249

250

251

252

253

254

255

256

257

258

259

260

261

262

263

264

265

266

267

268

269

270

271

measurement (Heinzelmann et al. 2011; Zheng et al. 2011). MiRNA-30a, as a member of the miRNA-30 family, has been correlated with several types of human cancers(Saleh et al. 2019; Sun et al. 2019; Wen et al. 2015b). The expression level of miRNA-30a was down-regulated in breast cancer(Zeng et al. 2013) but up-regulated in prostate cancer and ovarian serous adenocarcinoma (Fredsøe et al. 2018; Zhou et al. 2015). Our present study revealed that miRNA30a in BALF was correlated with different stages of lung cancer. The opposite activity of miR30a in various cancer type and different stages in one cancer type indicated that the biological function of miR-30a might be complicated and associated with a specific cancer type and tumor microenvironment, which needs to be further explored.

There are several potential mechanism of miRNA-30a in the development, invasion and metastasis of lung cancer. First, miRNA-30a inhibits the epithelial-to-mesenchymal transition by targeting SNAI1, and thus, its downregulation in lung cancer results in cancer invasion and metastasis (Kumarswamy et al. 2012). Second, Guan, Y., et al. have found that miR-30a suppresses lung cancer progression by targeting SIRT1 (Guan et al. 2017). In addition, expression of miR-30a inhibitor the growth of lung cancer cells by targeting MEF2D (Luan et al. 2018). Further studies are necessary to identify the target genes of miR-30a and elucidate the underlying mechanism that regulates the biogenesis of miR-30a.

The expression level of miRNA-30a in body fluids, including BALF and the plasma(Liang et al. 2019) of lung cancer patients, were significantly upregulated, while the opposite trend was observed in lung cancer tissue(Boeri et al. 2011; Kumarswamy et al. 2012; Tang et al. 2015). There are several possible mechanisms. microRNAs are transported into the extracellular fluid 
272 environment from cells by exosomes and microvesicles (Etheridge et al. 2011). Pritchard et al.

273 have presented evidence showing that blood cells are a major contributor to circulating miRNAs

274 (Pritchard et al. 2012). The extracellular circulation of miRNAs in lung cancer patients may be

275 due to a response of blood cells to the tumour state (Patnaik et al. 2012) and shifting to various

276 body fluids. However, the exact mechanism still needs to be investigated. However, the exact

277 biological basis of this discrepancy between miRNA-30a expression changes in cancer tissues

278 and in circulating body fluids in lung cancer still remains unclear and needs to be investigated in

279 the future research.

280

281

The present study does have some limitations. First, as a hospital-based case-control study, the

282

283

284

285

286

287

288

289

290

291

292

293

294

relatively small sample size of each group was unavoidable. Future studies with larger cohorts

are warranted to validate these findings. Second, there was no significance in BALF miRNA-30a

expression levels among the adenocarcinoma, squamous cell carcinoma and small cell lung

cancer, and it may be related to the relative small sample size of small cell lung cancer. Third the

underlying molecular mechanism that accounts for the contradiction between miRNA-30a

expression in cancer tissues and in circulating body fluids of lung cancer needs further

investigation.

\section{Conclusions}

In conclusion, BALF miRNA-30a was significantly up-regulated in lung cancer patients and correlated with early stage lung cancer. These present findings indicated that BALF miRNA-30a relative expression level could be used as a biomarker in early detection of lung cancer for individuals undergoing FB but with invisible tumor in the bronchial tree or risk factors of biopsy.

Peer] reviewing PDF | (2019:12:43901:1:1:NEW 9 Mar 2021) 


\section{Acknowledgements}

297 The authors thank Prof Huajing Wan for advising on the article revision. The authors thank the 298 patients who took part in the study and the staff of the Department of Pulmonary and Critical 299 Care Medicine, West China Hospital, Sichuan University for their help with data collection. 
References

303

Alberg AJ, Brock MV, Ford JG, Samet JM, and Spivack SD. 2013. Epidemiology of lung cancer: Diagnosis and management of lung cancer, 3rd ed: American College of Chest Physicians evidence-based clinical practice guidelines. Chest 143:e1S-e29S. 10.1378/chest.12-2345

Bartel DP. 2004. MicroRNAs: genomics, biogenesis, mechanism, and function. cell.

Berezikov E, van Tetering G, Verheul M, van de Belt J, van Laake L, Vos J, Verloop R, van de Wetering M, Guryev V, Takada S, van Zonneveld AJ, Mano H, Plasterk R, and Cuppen E. 2006. Many novel mammalian microRNA candidates identified by extensive cloning and RAKE analysis. Genome Res 16:1289-1298. 10.1101/gr.5159906

Boehm M, and Slack F. 2005. A developmental timing microRNA and its target regulate life span in C. elegans. Science.

Boeri M, Verri C, Conte D, Roz L, Modena P, Facchinetti F, Calabro E, Croce CM, Pastorino U, and Sozzi G. 2011. MicroRNA signatures in tissues and plasma predict development and prognosis of computed tomography detected lung cancer. Proc Natl Acad Sci U S A 108:3713-3718. 10.1073/pnas.1100048108

Bridge G, Monteiro R, Henderson S, Emuss V, Lagos D, Georgopoulou D, Patient R, and Boshoff C. 2012. The microRNA-30 family targets DLL4 to modulate endothelial cell behavior during angiogenesis. Blood 120:5063-5072. 10.1182/blood-2012-04-423004

Bussing I, Slack FJ, and Grosshans H. 2008. let-7 microRNAs in development, stem cells and cancer. Trends Mol Med 14:400-409. 10.1016/j.molmed.2008.07.001

Chen X, Ba Y, Ma L, Cai X, Yin Y, Wang K, Guo J, Zhang Y, Chen J, Guo X, Li Q, Li X, Wang W, Zhang Y, Wang J, Jiang X, Xiang Y, Xu C, Zheng P, Zhang J, Li R, Zhang H, Shang X, Gong T, Ning G, Wang J, Zen K, Zhang J, and Zhang CY. 2008. Characterization of microRNAs in serum: a novel class of biomarkers for diagnosis of cancer and other diseases. Cell Res 18:997-1006. 10.1038/cr.2008.282

Dela Cruz CS, Tanoue LT, and Matthay RA. 2011. Lung cancer: epidemiology, etiology, and prevention. Clin Chest Med 32:605-644. 10.1016/j.ccm.2011.09.001

Du Rand IA, Blaikley J, Booton R, Chaudhuri N, Gupta V, Khalid S, Mandal S, Martin J, Mills J, Navani N, Rahman NM, Wrightson JM, Munavvar M, and British Thoracic Society Bronchoscopy Guideline G. 2013. British Thoracic Society guideline for diagnostic flexible bronchoscopy in adults: accredited by NICE. Thorax 68 Suppl 1:i1-i44. 10.1136/thoraxjnl-2013-203618

Esquela-Kerscher A, and Slack FJ. 2006. Oncomirs - microRNAs with a role in cancer. Nat Rev Cancer 6:259-269. $10.1038 / \mathrm{nrc1840}$

Etheridge A, Lee I, Hood L, Galas D, and Wang K. 2011. Extracellular microRNA: A new source of biomarkers. Mutation Research/Fundamental and Molecular Mechanisms of Mutagenesis 717:85-90. 10.1016/j.mrfmmm.2011.03.004

Fredsøe J, Rasmussen AKI, Thomsen AR, Mouritzen P, Høyer S, Borre M, Ørntoft TF, and Sørensen KD. 2018. Diagnostic and Prognostic MicroRNA Biomarkers for Prostate Cancer in Cell-free Urine. Eur Urol Focus 4:825-833. 10.1016/j.euf.2017.02.018

Guan Y, Rao Z, and Chen C. 2017. miR-30a suppresses lung cancer progression by targeting SIRT1. Oncotarget Dec 21;9(4):4924-4934.

Heinzelmann J, Henning B, Sanjmyatav J, Posorski N, Steiner T, Wunderlich H, Gajda MR, and Junker K. 2011. Specific miRNA signatures are associated with metastasis and poor prognosis in clear cell renal cell carcinoma. World J Urol 29:367-373. 10.1007/s00345-010-0633-4

Johnson CD, Esquela-Kerscher A, Stefani G, Byrom M, Kelnar K, Ovcharenko D, Wilson M, Wang X, Shelton J, Shingara J, Chin L, Brown D, and Slack FJ. 2007. The let-7 microRNA represses cell proliferation pathways in human cells. Cancer Res 67:7713-7722. 10.1158/0008-5472.CAN-07-1083

Kim JO, Gazala S, Razzak R, Guo L, Ghosh S, Roa WH, and Bedard EL. 2015. Non-small cell lung cancer detection using microRNA expression profiling of bronchoalveolar lavage fluid and sputum. ANTICANCER RESEARCH.

Kumarswamy R, Mudduluru G, Ceppi P, Muppala S, Kozlowski M, Niklinski J, Papotti M, and Allgayer H. 2012. 
MicroRNA-30a inhibits epithelial-to-mesenchymal transition by targeting Snai1 and is downregulated in non-small cell lung cancer. Int J Cancer 130:2044-2053. 10.1002/ijc.26218

Liang LB, Zhu WJ, Chen XM, and Luo FA-O. 2019. Plasma miR-30a-5p as an early novel noninvasive diagnostic and prognostic biomarker for lung cancer. Future Oncol (2019) 15(32):3711-3721.

Luan N, Wang Y, and Liu X. 2018. Absent expression of miR-30a promotes the growth of lung cancer cells by targeting MEF2D. Oncol Lett Jul;16(1):1173-1179.

Mao L, Liu S, Hu L, Jia L, Wang H, Guo M, Chen C, Liu Y, and Xu L. 2018. miR-30 Family: A Promising Regulator in Development and Disease. Biomed Res Int 2018:9623412. 10.1155/2018/9623412

McLean AN, Semple PA, Franklin DH, Petrie G, Millar EA, and Douglas JG. 1998. The Scottish multi-centre prospective study of bronchoscopy for bronchial carcinoma and suggested audit standards. Respir Med 92:1110-1115. 10.1016/s0954-6111(98)90403-6

Mitchell PS, Parkin Rk Fau - Kroh EM, Kroh Em Fau - Fritz BR, Fritz Br Fau - Wyman SK, Wyman Sk Fau - PogosovaAgadjanyan EL, Pogosova-Agadjanyan El Fau - Peterson A, Peterson A Fau - Noteboom J, Noteboom J Fau O'Briant KC, O'Briant Kc Fau - Allen A, Allen A Fau - Lin DW, Lin Dw Fau - Urban N, Urban N Fau - Drescher CW, Drescher Cw Fau - Knudsen BS, Knudsen Bs Fau - Stirewalt DL, Stirewalt DI Fau - Gentleman R, Gentleman R Fau - Vessella RL, Vessella RI Fau - Nelson PS, Nelson Ps Fau - Martin DB, Martin Db Fau Tewari M, and Tewari M. 2008. Circulating microRNAs as stable blood-based markers for cancer detection. PNAS.

Patnaik SK, Yendamuri S, Kannisto E, Kucharczuk JC, Singhal S, and Vachani A. 2012. MicroRNA expression profiles of whole blood in lung adenocarcinoma. PLoS One 7:e46045. 10.1371/journal.pone.0046045

Pritchard CC, Kroh E, Wood B, Arroyo JD, Dougherty KJ, Miyaji MM, Tait JF, and Tewari M. 2012. Blood cell origin of circulating microRNAs: a cautionary note for cancer biomarker studies. Cancer Prev Res (Phila) 5:492-497. 10.1158/1940-6207.CAPR-11-0370

Rivera MP, and Mehta AC. 2007. Initial diagnosis of lung cancer: ACCP evidence-based clinical practice guidelines (2nd edition). Chest 132:131s-148s. 10.1378/chest.07-1357

Saleh AD, Cheng H, Martin SE, Si H, Ormanoglu P, Carlson S, Clavijo PE, Yang X, Das R, Cornelius S, Couper J, Chepeha D, Danilova L, Harris TM, Prystowsky MB, Childs GJ, Smith RV, Robertson AG, Jones SJM, Cherniack AD, Kim SS, Rait A, Pirollo KF, Chang EH, Chen Z, and Van Waes C. 2019. Integrated Genomic and Functional microRNA Analysis Identifies miR-30-5p as a Tumor Suppressor and Potential Therapeutic Nanomedicine in Head and Neck Cancer. Clin Cancer Res 25:2860-2873. 10.1158/1078-0432.Ccr-18-0716

Sun L, Chen Y, Su Q, Tang X, Liang Y, Che G, and Luo F. 2016. Increased Plasma miRNA-30a as a Biomarker for NonSmall Cell Lung Cancer. Medical Science Monitor 22:647-655. 10.12659/msm.897330

Sun Y, Yang B, Lin M, Yu H, Chen H, and Zhang Z. 2019. Identification of serum miR-30a-5p as a diagnostic and prognostic biomarker in colorectal cancer. Cancer Biomark 24:299-305. 10.3233/cbm-182129

Tang R, Liang L, Luo D, Feng Z, Huang Q, He R, Gan T, Yang L, and Chen G. 2015. Downregulation of MiR-30a is Associated with Poor Prognosis in Lung Cancer. Med Sci Monit 21:2514-2520. 10.12659/msm.894372

Tissot C, Toffart AC, Villar S, Souquet PJ, Merle P, Moro-Sibilot D, Perol M, Zavadil J, Brambilla C, Olivier M, and Couraud S. 2015. Circulating free DNA concentration is an independent prognostic biomarker in lung cancer. Eur Respir J 46:1773-1780. 10.1183/13993003.00676-2015

Torre LA, Siegel RL, and Jemal A. 2016. Lung Cancer Statistics. Lung Cancer and Personalized Medicine, 1-19.

Tsujiura M, Ichikawa D, Komatsu S, Shiozaki A, Takeshita H, Kosuga T, Konishi H, Morimura R, Deguchi K, Fujiwara $\mathrm{H}$, Okamoto K, and Otsuji E. 2010. Circulating microRNAs in plasma of patients with gastric cancers. Br J Cancer 102:1174-1179. 10.1038/sj.bjc.6605608

Wen XP, Ma HL, Zhao LY, Zhang W, and Dang CX. 2015a. MiR-30a suppresses non-small cell lung cancer progression through AKT signaling pathway by targeting IGF1R. Cell Mol Biol 61 (2):78-85.

Wen Y, Han J, Chen J, Dong J, Xia Y, Liu J, Jiang Y, Dai J, Lu J, Jin G, Han J, Wei Q, Shen H, Sun B, and Hu Z. 2015b. Plasma miRNAs as early biomarkers for detecting hepatocellular carcinoma. Int J Cancer 137:1679-1690. 10.1002/ijc. 29544

Yao J, Liang L, Huang S, Ding J, Tan N, Zhao Y, Yan M, Ge C, Zhang Z, Chen T, Wan D, Yao M, Li J, Gu J, and He X. 2010. MicroRNA-30d promotes tumor invasion and metastasis by targeting Galphai2 in hepatocellular carcinoma. Hepatology 51:846-856. 10.1002/hep.23443

Yi R, Qin Y, Macara IG, and Cullen BR. 2003. Exportin-5 mediates the nuclear export of pre-microRNAs and short hairpin RNAs. Genes Dev 17:3011-3016. 10.1101/gad.1158803

PeerJ reviewing PDF | (2019:12:43901:1:1:NEW 9 Mar 2021) 
403

Zeng RC, Zhang W, Yan XQ, Ye ZQ, Chen ED, Huang DP, Zhang XH, and Huang GL. 2013. Down-regulation of miRNA30a in human plasma is a novel marker for breast cancer. Med Oncol 30:477. 10.1007/s12032-013-0477-z

Zheng D, Haddadin S Fau - Wang Y, Wang Y Fau - Gu L-Q, Gu Lq Fau - Perry MC, Perry Mc Fau - Freter CE, Freter Ce Fau - Wang MX, and Wang MX. 2011. Plasma microRNAs as novel biomarkers for early detection of lung cancer. Int J Clin Exp Pathol 4(6):575-586.

Zhou J, Gong G, Tan H, Dai F, Zhu X, Chen Y, Wang J, Liu Y, Chen P, Wu X, and Wen J. 2015. Urinary microRNA-30a$5 p$ is a potential biomarker for ovarian serous adenocarcinoma. Oncol Rep 33:2915-2923. 10.3892/or.2015.3937

\section{Figure legends}

Fig. 1 miRNA-30a levels in BALF samples obtained from lung cancer patients, patients with benign lung diseases and healthy control, as determined by quantitative real-time polymerase chain reaction (RT-qPCR) $(\mathrm{n}=55$ in the benign control group, $\mathrm{n}=99$ in the lung cancer group and $\mathrm{n}=20$ in the healthy control). ${ }^{*} \mathrm{p}<0.001$. Data was $\log$ transformed to approximate a normal distribution.

Fig. 2 miRNA-30a levels in BALF specimens obtained from the sides with and without lung cancer lesions $(n=20, p>0.05)$

Fig. 3 Bronchoalveolar lavage fluid (BALF) miRNA-30a expression was measured by quantitative real-time polymerase chain reaction $(\mathrm{RT}-\mathrm{qPCR})(\mathrm{n}=22$ for stages $\mathrm{I}-\mathrm{IIA}$ and $\mathrm{n}=62$ for stages IIB-IV). ${ }^{*} \mathrm{p}<0.05$. Data was $\log$ transformed to approximate a normal distribution.

Fig. 4 Receiver operating characteristic (ROC) curve analysis of miRNA-30a level to distinguish between lung cancer and benign lung diseases. The area under the curve for miRNA-30a was 0.822 , and the Youden index was 0.499. 
431 Fig. 5 miRNA-30a levels in stages I-IIA lung cancer patients $(n=22)$ and benign controls $(n=55)$,

432 as determined by quantitative real-time polymerase chain reaction (RT-qPCR). ${ }^{*} \mathrm{p}<0.001$. Data

433 was $\log$ transformed to approximate a normal distribution.

434

435 Fig. 6 Receiver operating characteristic (ROC) curve analysis of miRNA-30a level to distinguish 436 between early lung cancer and benign lung diseases. The area under the curve for miRNA-30a 437 was 0.882 (95\% confidence interval: $0.806-0.958, \mathrm{p}<0.001)$, and the Youden index was 0.654.

Fig. 7 Receiver operating characteristic (ROC) curve analysis of miRNA-30a level to distinguish between stages I-IIA adenocarcinoma and benign lung diseases. The area under the curve for miRNA-30a was 0.875 (95\% confidence interval: $0.790-0.960, \mathrm{p}<0.001)$.

442

Fig. 8 Receiver operating characteristic (ROC) curve analysis of miRNA-30a level, CEA level, and a combination of miRNA-30a and carcinoembryonic antigen (CEA) levels to distinguish between stages I-IIA adenocarcinoma and benign lung diseases. The area under the curve for

446 miRNA-30a was $0.910(95 \%$ confidence interval: $0.808-1.000, \mathrm{p}<0.001)$. 


\section{Table $\mathbf{1}$ (on next page)}

Table 1. Demographic characteristics of study subjects 
1

2 Table 1. Demographic characteristics of study subjects

\begin{tabular}{lllll}
\hline & $\begin{array}{l}\text { Lung cancer cases } \\
(\mathrm{n}=99)\end{array}$ & $\begin{array}{l}\text { Benign control cases } \\
(\mathrm{n}=55)\end{array}$ & $\begin{array}{l}\text { Healthy control } \\
(\mathrm{n}=20)\end{array}$ & p-value \\
$\begin{array}{l}\text { Sex } \\
\text { male: female }\end{array}$ & $62: 37$ & $39: 16$ & $12: 8$ & 0.52 \\
Median age(y) & $57.97 \pm 10.33$ & $54.53 \pm 10.62$ & $53.05 \pm 13.20$ & 0.061 \\
BMI, $\mathrm{kg} \cdot \mathrm{m}^{-2}$ & $22.77 \pm 2.80$ & $23.11 \pm 3.97$ & $22.55 \pm 1.94$ & 0.728 \\
Smoking status & & & & \\
Smoker & $56(57 \%)$ & $26(47 \%)$ & $9(45 \%)$ & 0.426 \\
Never smoked & $43(43 \%)$ & $29(53 \%)$ & $11(55 \%)$ & \\
\hline
\end{tabular}

4 BMI: body mass index. 


\section{Table 2 (on next page)}

Table 2. Relationship between miRNA-30a levels and clinicopathological features among patients with lung cancer 
1 Table 2. Relationship between miRNA-30a levels and clinicopathological features among

2 patients with lung cancer

\section{Clinicopathological features Subgroup Subjects miRNA-30a relative expression}

$$
\text { Mean } \pm \text { SD } \quad \text { p-value }
$$

Tissue type

SCLC

15

$0.72 \pm 0.58$

NSCLC

84

$0.74 \pm 0.55$

0.911

Tissue type subgroup

SCC

22

$0.71 \pm 0.44$

ADC

60

$0.76 \pm 0.59$

SCLC

15

$0.72 \pm 0.58$

0.924

Age, years

$\leq 60$

51

$0.69 \pm 0.55$

$>60$

48

$0.79 \pm 0.55$

Sex

Male

62

$0.75 \pm 0.58$

Female $\quad 37$

$0.71 \pm 0.50$

0.710

Smoking

Yes

56

$0.77 \pm 0.59$

No

43

$0.69 \pm 0.49$

0.473

Clinical TNM stage

I-IIA

22

$0.98 \pm 0.64$

IIB-IV

62

$0.66 \pm 0.54 \quad 0.025^{*}$

3 SD, standard deviation; SCLC, small-cell lung cancer; NSCLC, non-small-cell lung cancer; SCC,

4 squamous cell carcinoma; ADC, adenocarcinoma.

$5 \quad *_{\mathrm{p}}<0.05$

6

7 


\section{Table 3 (on next page)}

Table 3. Diagnostic value of bronchoalveolar lavage fluid miRNA-30a based on the optimal cut-off point 
1 Table 3. Diagnostic value of bronchoalveolar lavage fluid miRNA-30a based on the optimal cut-

2 off point

Value $\quad 95 \%$ CI

\section{Cut-off point}

TPR

TNR

LR+

LR-

PPV

NPV

2.0316

$80.8 \%$

$69.1 \%$

2.61

0.27

$82.4 \%$

$66.6 \%$
$0.7140-0.8776$

$0.5503-0.8047$

$1.7409-3.9260$

$0.1824-0.4228$

$0.7313-0.8917$

$0.5283-0.7825$

$3 \mathrm{CI}$, confidence interval; TPR, true positive rate (sensitivity); TNR, true negative rate

4 (specificity); LR+, positive likelihood ratio; LR-, negative likelihood ratio; PPV, positive

5 predictive value; NPV, negative predictive value. 


\section{Table 4 (on next page)}

Table 4. Diagnostic value of bronchoalveolar lavage fluid miRNA-30a in early (stages I-IIA) lung cancer 
1 Table 4. Diagnostic value of bronchoalveolar lavage fluid miRNA-30a in early (stages I-IIA)

2 lung cancer

\begin{tabular}{lll}
\hline & Value & $95 \%$ CI \\
\hline Cut-off point & 2.6508 & $0.69-0.98$ \\
TPR & $90.9 \%$ & $0.60-0.845$ \\
TNR & $74.5 \%$ & $2.23-5.72$ \\
LR+ & 3.57 & $0.03-0.46$ \\
LR- & 0.12 & $0.41-0.75$ \\
PPV & $58.8 \%$ & $0.83-0.99$ \\
NPV & $95.3 \%$ &
\end{tabular}

$3 \mathrm{CI}$, confidence interval; TPR, true positive rate (sensitivity); TNR, true negative rate

4 (specificity); LR+, positive likelihood ratio; LR-, negative likelihood ratio; PPV, positive

5 predictive value; NPV, negative predictive value.

6 


\section{Table 5 (on next page)}

Table 5. Diagnostic value of bronchoalveolar lavage fluid miRNA-30a for different pathologic types of lung cancer 
1 Table 5. Diagnostic value of bronchoalveolar lavage fluid miRNA-30a for different pathologic

2 types of lung cancer

\begin{tabular}{lcccc}
\hline Category & Subjects & AUC & p-value & $95 \%$ CI \\
\hline ADC versus control & $60: 55$ & 0.821 & $<0.001$ & $0.75-0.90$ \\
SCC versus control & $22: 55$ & 0.832 & $<0.001$ & $0.74-0.92$ \\
SCLC versus control & $15: 55$ & 0.817 & $<0.001$ & $0.71-0.93$ \\
NSCLC versus control & $84: 55$ & 0.823 & $<0.001$ & $0.75-0.89$
\end{tabular}

3 AUC, area under the receiver operating characteristic curve; CI, confidence interval; ADC,

4 adenocarcinoma; SCC, squamous cell carcinoma; SCLC, small-cell lung cancer; NSCLC, non-

5 small-cell lung cancer.

6 Control group (benign disease, $\mathrm{n}=55$ ). 
Table 6(on next page)

Table 6. Diagnostic value of bronchoalveolar lavage fluid miRNA-30a in different pathologic types and TNM stages of lung cancer 
1 Table 6. Diagnostic value of bronchoalveolar lavage fluid miRNA-30a in different pathologic

2 types and TNM stages of lung cancer

\begin{tabular}{lcccc}
\hline Tissue type & Subjects & AUC & p-value & $95 \%$ CI \\
\hline ADC, stages I-IIA & 18 & 0.875 & $<0.001$ & $0.79-0.96$ \\
NSCLC, stages I-IIA & 21 & 0.876 & $<0.001$ & $0.80-0.96$ \\
SCC, stages IIB-IV & 14 & 0.822 & $<0.001$ & $0.72-0.93$ \\
SCLC, stages IIB-IV & 10 & 0.773 & $<0.05$ & $0.63-0.91$ \\
ADC, stages IIB-IV & 37 & 0.783 & $<0.001$ & $0.69-0.87$ \\
NSCLC, stages IIB-IV & 52 & 0.796 & $<0.001$ & $0.71-0.88$
\end{tabular}

3 AUC, area under the receiver operating characteristic curve; CI, confidence interval; ADC,

4 adenocarcinoma; NSCLC, non-small-cell lung cancer; SCC, squamous cell carcinoma; SCLC,

5 small-cell lung cancer.

6

7 


\section{Table 7 (on next page)}

Table 7. Diagnostic value of bronchoalveolar lavage fluid miRNA-30a in combination with plasma tumor markers 
1 Table 7. Diagnostic value of bronchoalveolar lavage fluid miRNA-30a in combination with 2 plasma tumor markers

\begin{tabular}{|c|c|c|c|c|c|}
\hline Diagnostic value & Subjects & Subgroup & AUC & $\mathrm{p}$-value & $95 \% \mathrm{CI}$ \\
\hline \multirow[t]{3}{*}{ ADC, stages I-IIA } & 10 & miR-30a & 0.910 & $<0.001$ & $0.81-1.00$ \\
\hline & & CEA & 0.572 & $>0.05$ & $0.36-0.78$ \\
\hline & & $\mathrm{CEA}+\mathrm{miR}-30 \mathrm{a}$ & 0.903 & $<0.001$ & $0.80-1.00$ \\
\hline \multirow[t]{3}{*}{ ADC, stages IIB-IV } & 29 & miR-30a & 0.790 & $<0.001$ & $0.68-0.91$ \\
\hline & & CEA & 0.840 & $<0.001$ & $0.74-0.94$ \\
\hline & & $\mathrm{CEA}+\mathrm{miR}-30 \mathrm{a}$ & 0.880 & $<0.001$ & $0.80-0.97$ \\
\hline \multirow[t]{3}{*}{ NSCLC, stages I-IIA } & 12 & miR-30a & 0.882 & $<0.001$ & $0.78-0.99$ \\
\hline & & Cyfra21-1 & 0.438 & $>0.05$ & $0.22-0.65$ \\
\hline & & Cyfra 21-1 + miR-30a & 0.885 & $<0.001$ & $0.78-0.99$ \\
\hline \multirow[t]{3}{*}{ NSCLC, stages IIB-IV } & 34 & miR-30a & 0.796 & $<0.001$ & $0.69-0.91$ \\
\hline & & Cyfra21-1 & 0.813 & $<0.001$ & $0.71-0.92$ \\
\hline & & Cyfra21-1+miR-30a & 0.868 & $<0.001$ & $0.78-0.96$ \\
\hline
\end{tabular}

3 AUC, area under the receiver operating characteristic curve; CI, confidence interval; CEA, 4 carcinoembryonic antigen; ADC, adenocarcinoma; NSCLC. non-small-cell lung cancer. 


\section{Figure 1}

miRNA-30a levels in BALF samples obtained from lung cancer patients, patients with benign lung diseases and healthy control

Fig. 1 miRNA-30a levels in BALF samples obtained from lung cancer patients, patients with benign lung diseases and healthy control, as determined by quantitative real-time polymerase chain reaction (RT-qPCR) ( $n=55$ in the benign control group, $n=99$ in the lung cancer group and $n=20$ in the healthy control). $* p<0.001$. Data was log transformed to approximate a normal distribution. 


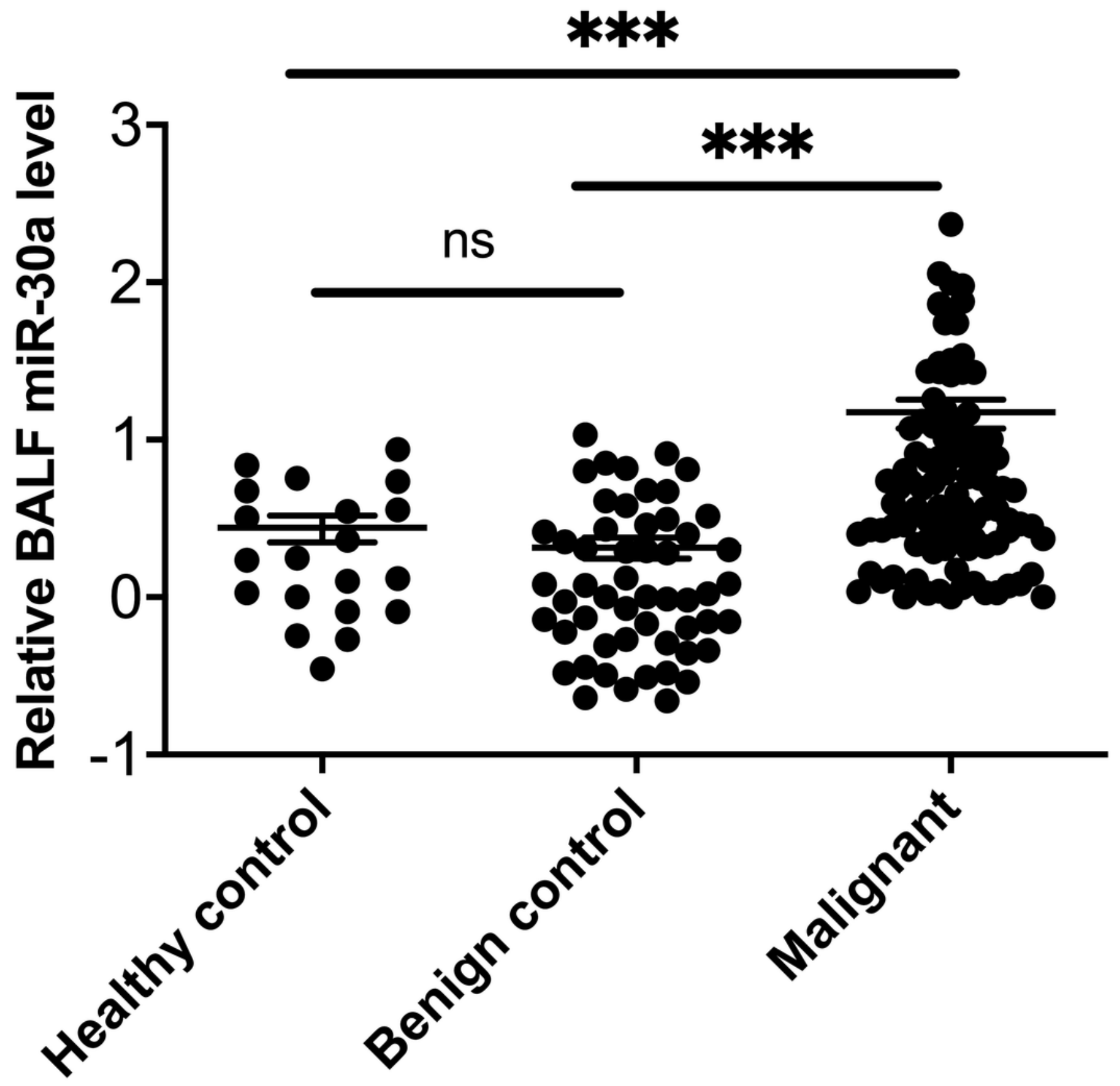


Figure 2

miRNA-30a levels in BALF specimens obtained from the sides with and without lung cancer lesions.

Fig. 2 miRNA-30a levels in BALF specimens obtained from the sides with and without lung cancer lesions $(n=20, p>0.05)$ 


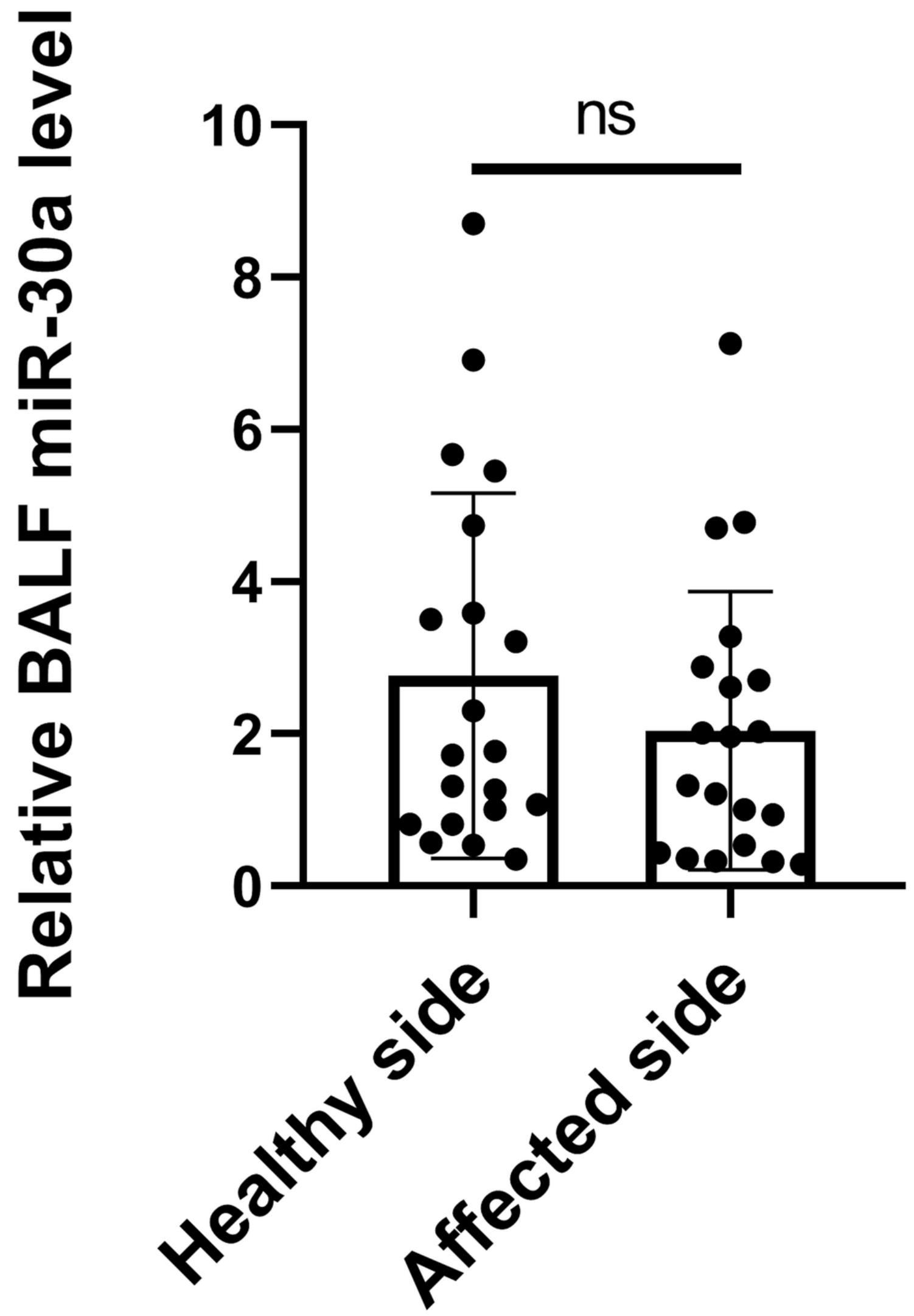


Figure 3

Correlation between BALF miRNA-30a levels and TNM stage

Fig. 3 Bronchoalveolar lavage fluid (BALF) miRNA-30a expression was measured by quantitative real-time polymerase chain reaction (RT-qPCR) ( $n=22$ for stages I-IIA and $n=62$ for stages IIB-IV). ${ }^{*} p<0.05$. Data was log transformed to approximate a normal distribution.

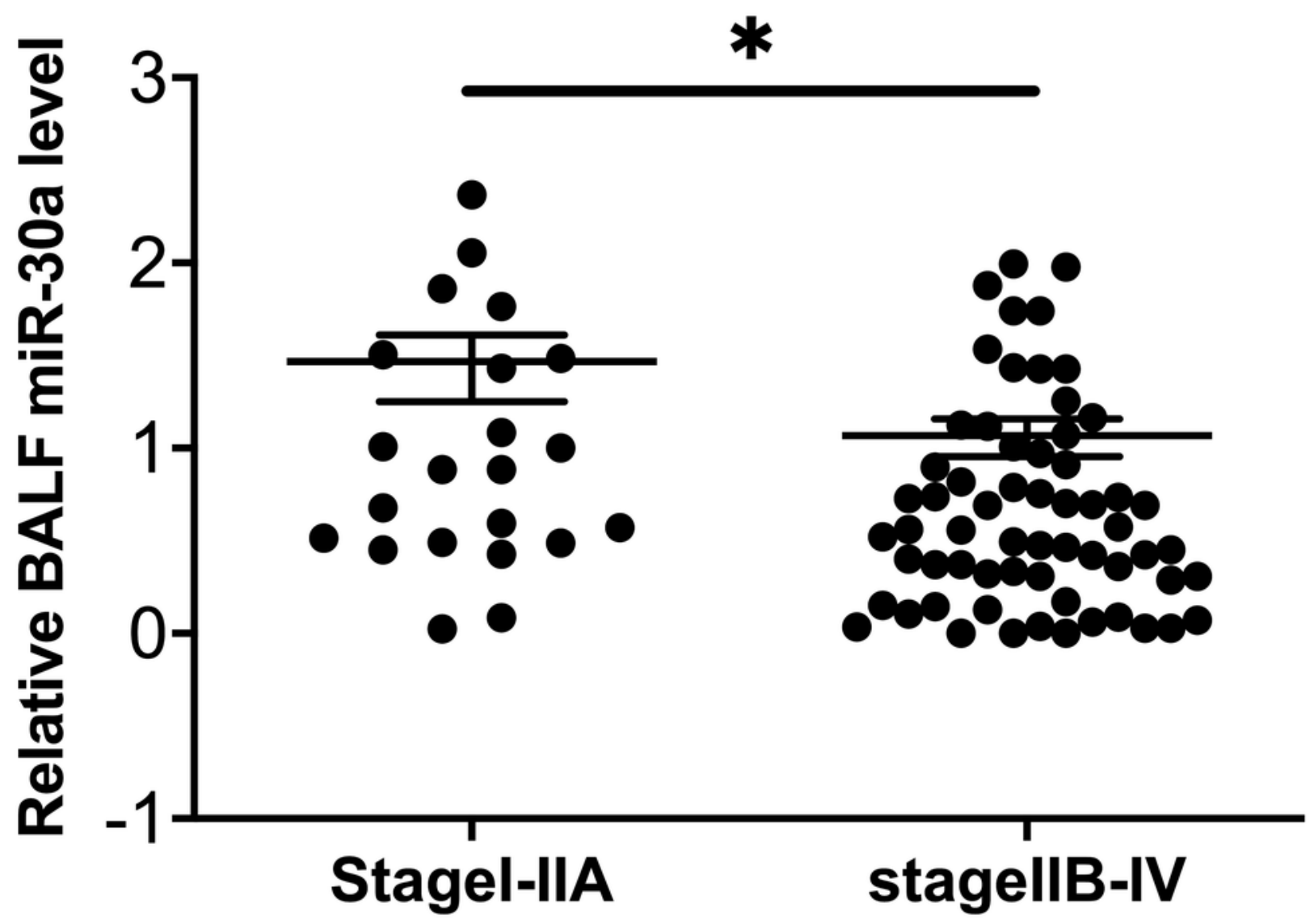


Figure 4

Receiver operating characteristic (ROC) curve analysis of miRNA-30a level to distinguish between lung cancer and benign lung diseases.

Fig. 4 Receiver operating characteristic (ROC) curve analysis of miRNA-30a level to distinguish between lung cancer and benign lung diseases. The area under the curve for miRNA-30a was 0.822, and the Youden index was 0.499 . 


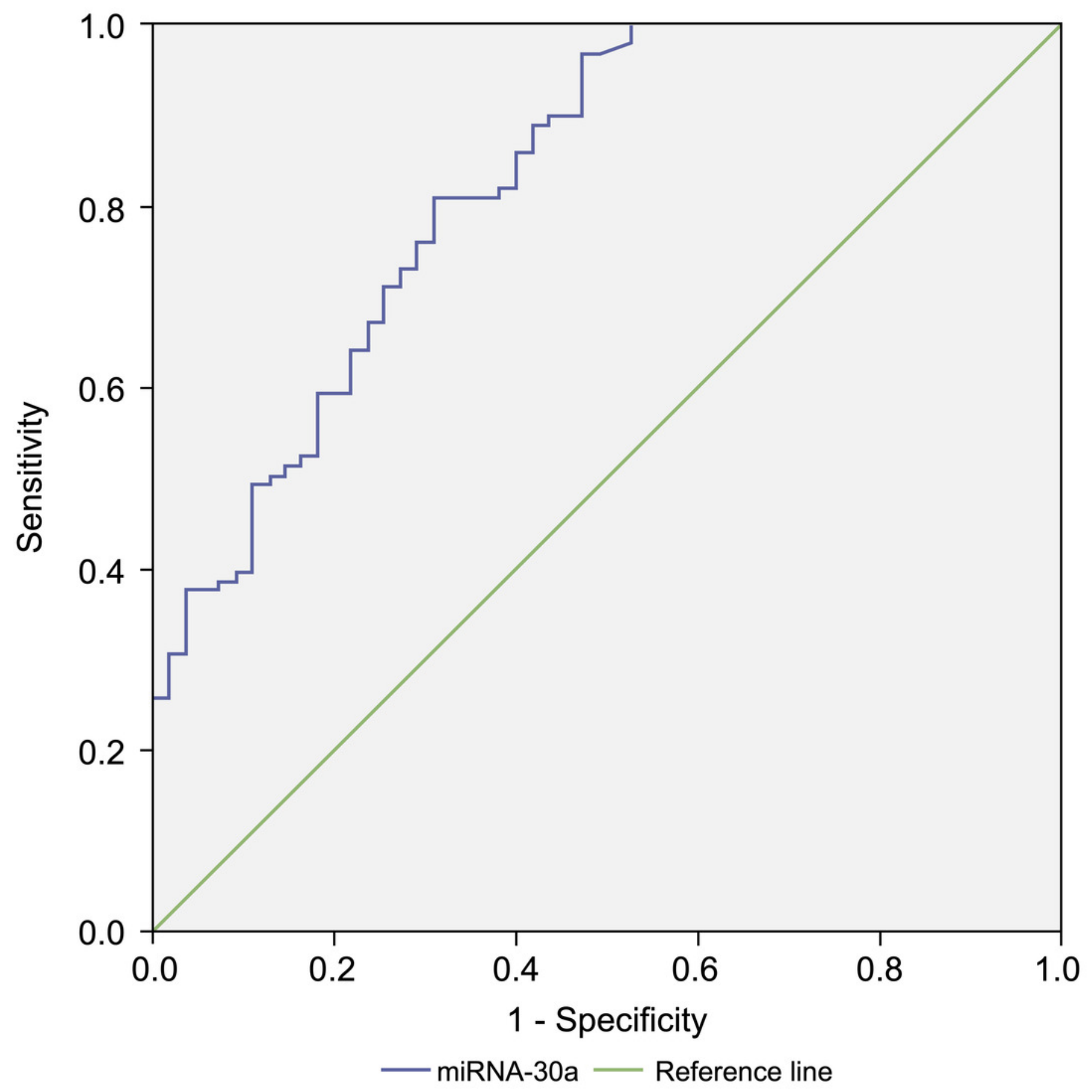


Figure 5

miRNA-30a levels in stages I-IIA lung cancer patients and benign controls.

Fig. 5 miRNA-30a levels in stages I-IIA lung cancer patients $(n=22)$ and benign controls $(n=55)$, as determined by quantitative real-time polymerase chain reaction (RT-qPCR). $* p<0.001$. Data was log transformed to approximate a normal distribution.

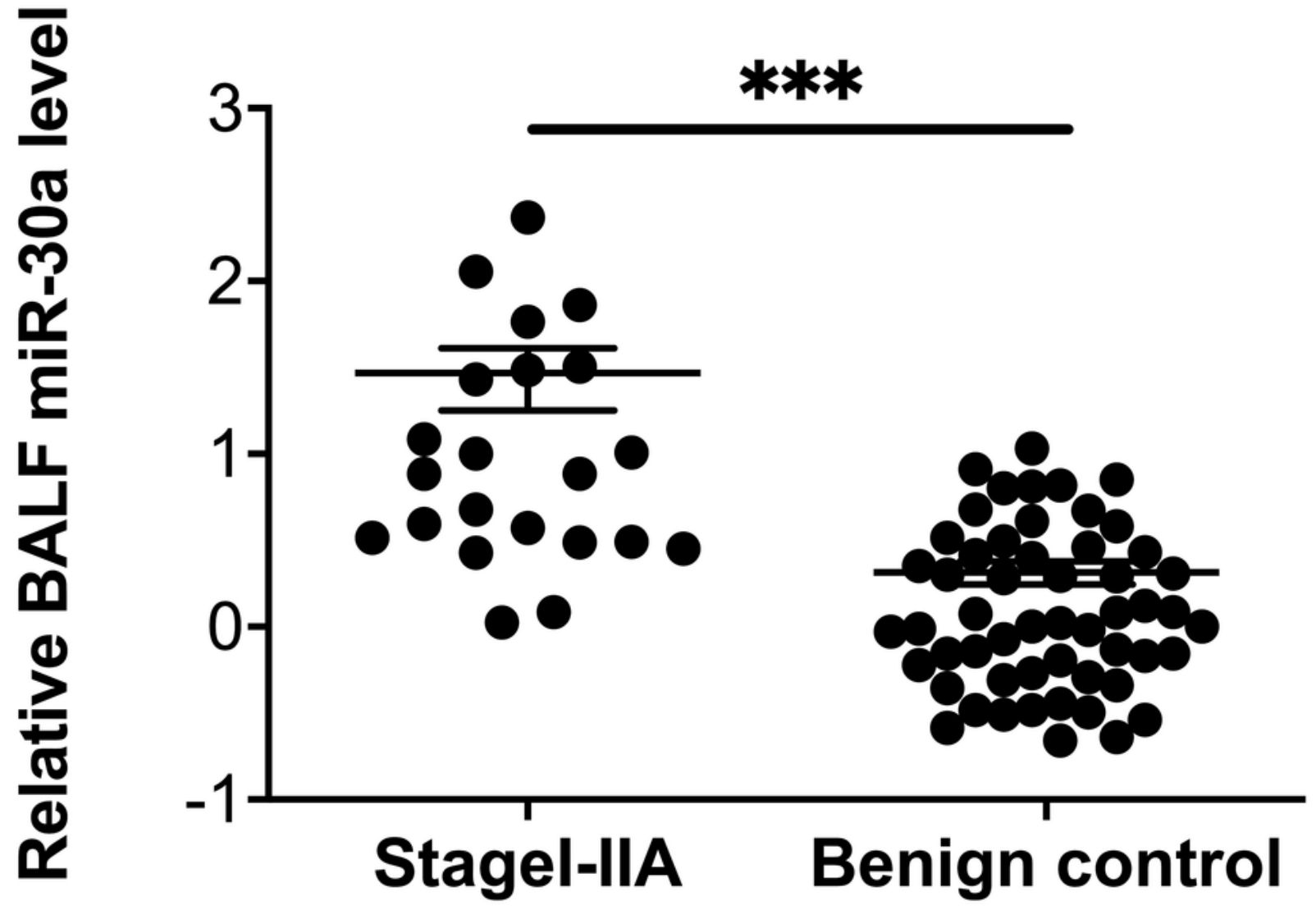




\section{Figure 6}

Receiver operating characteristic (ROC) curve analysis of miRNA-30a level to distinguish between early lung cancer and benign lung diseases.

Fig. 6 Receiver operating characteristic (ROC) curve analysis of miRNA-30a level to distinguish between early lung cancer and benign lung diseases. The area under the curve for miRNA-30a was 0.882 (95\% confidence interval: 0.806-0.958, $p<0.001$ ), and the Youden index was 0.654 . 


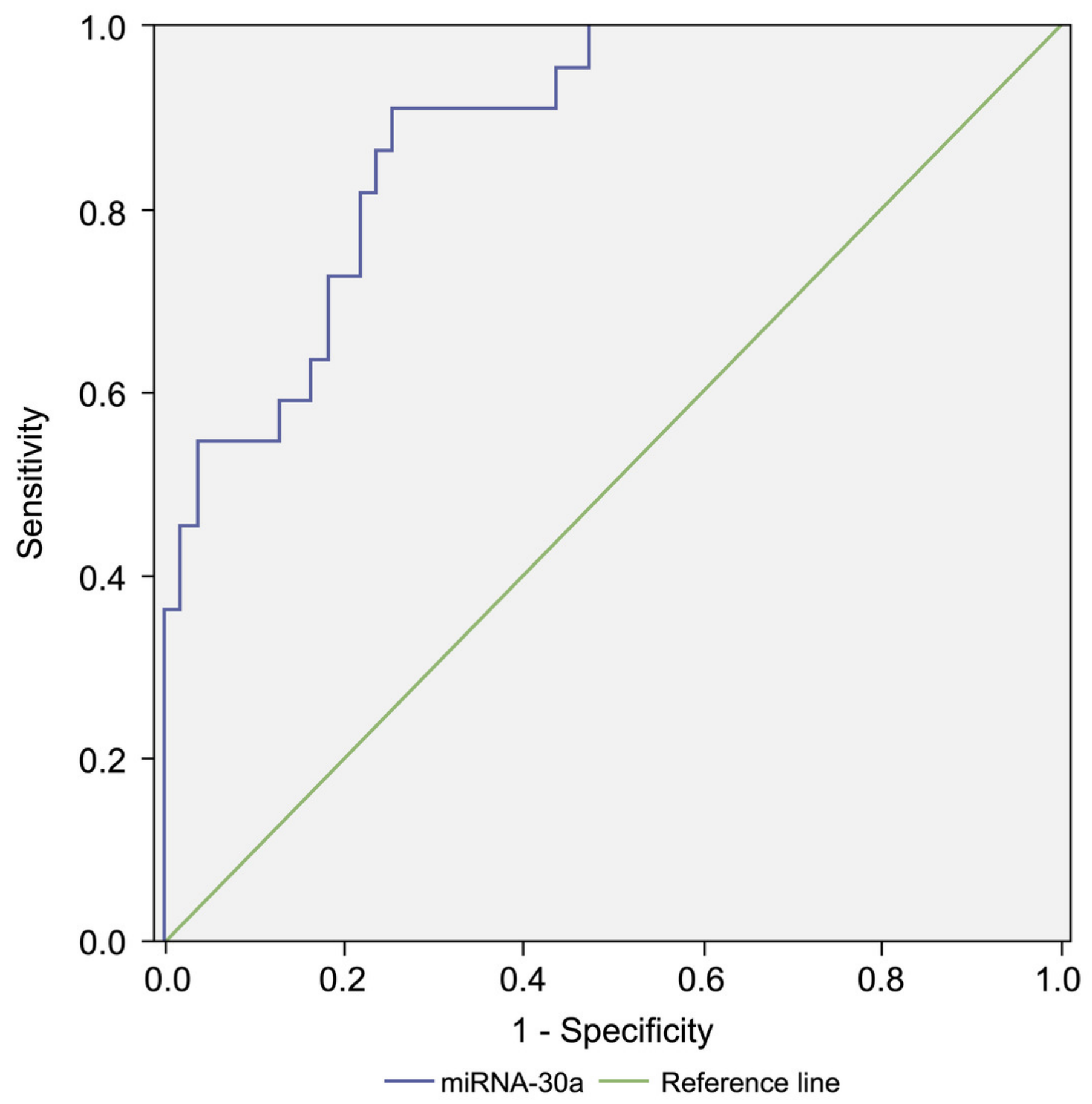




\section{Figure 7}

Receiver operating characteristic (ROC) curve analysis of miRNA-30a level to distinguish between stages I-IIA adenocarcinoma and benign lung diseases.

Fig. 7 Receiver operating characteristic (ROC) curve analysis of miRNA-30a level to distinguish between stages I-IIA adenocarcinoma and benign lung diseases. The area under the curve for miRNA-30a was 0.875 (95\% confidence interval: 0.790-0.960, $p<0.001$ ). 


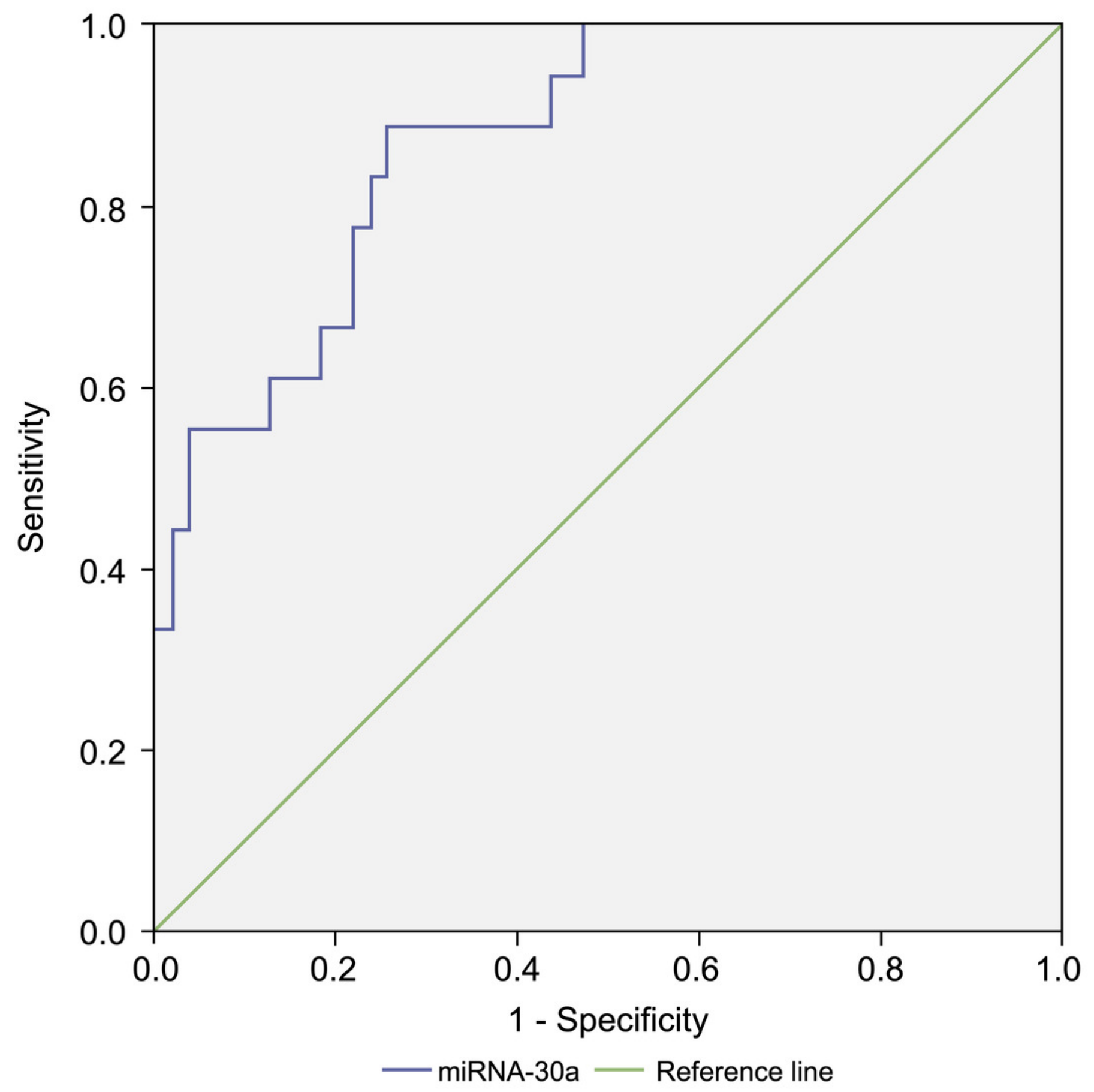




\section{Figure 8}

Receiver operating characteristic (ROC) curve analysis

Fig. 8 Receiver operating characteristic (ROC) curve analysis of miRNA-30a level, CEA level, and a combination of miRNA-30a and carcinoembryonic antigen (CEA) levels to distinguish between stages I-IIA adenocarcinoma and benign lung diseases. The area under the curve for miRNA-30a was 0.910 (95\% confidence interval: $0.808-1.000, p<0.001$ ). 


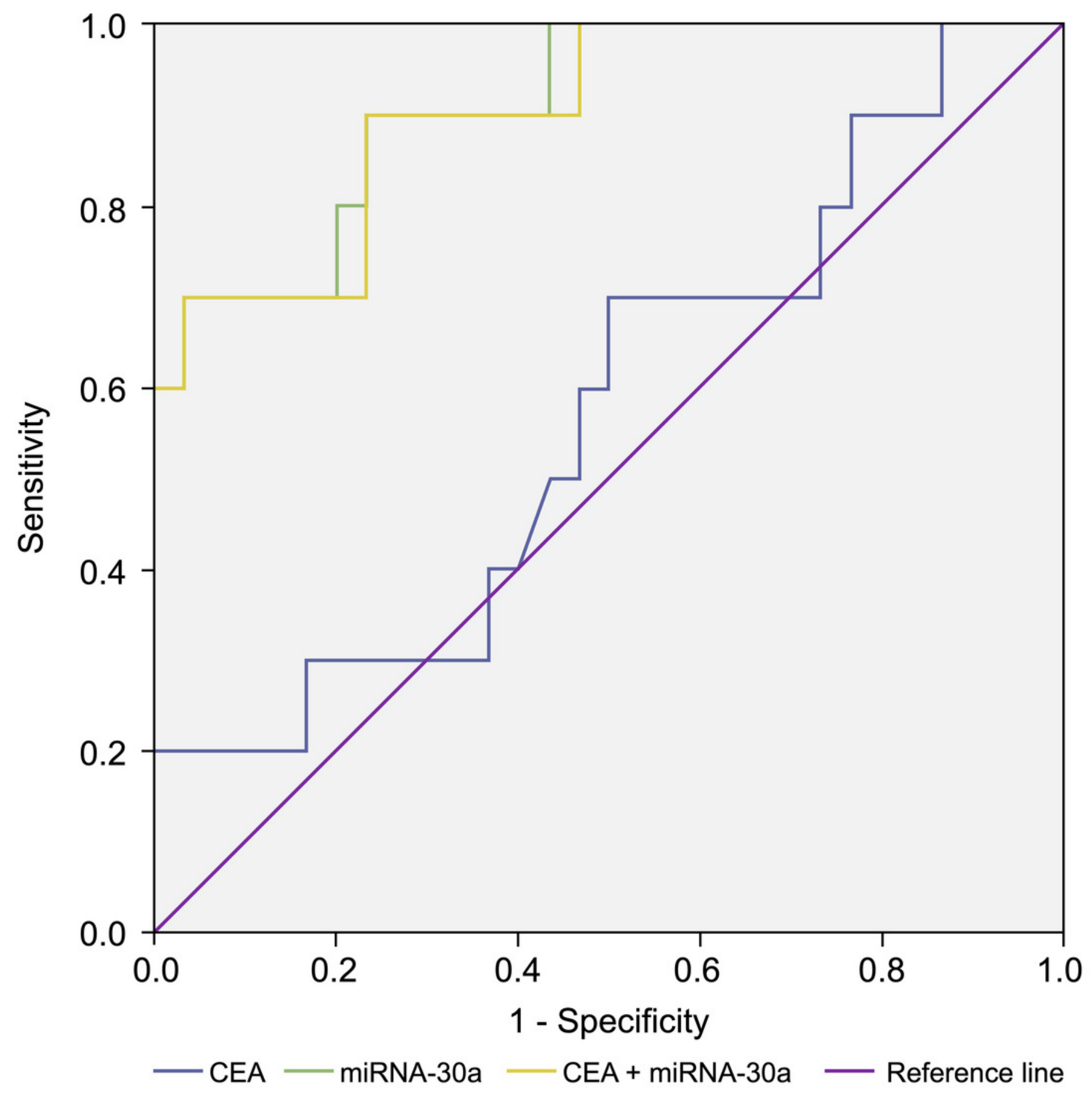

